\title{
Review: oral antifungal drugs promote cure of fungal infections of the foot
}

Bell-Syer SE, Hart R, Crawford F, et al. Oral treatments for fungal infections of the skin of the foot. Cochrane Database Syst Rev 2002;(2):CD003584 (latest version 5 Dec 2001).

\section{QUESTION: In patients with fungal infections of the skin of the foot (tinea pedis), does oral treatment eradicate the infection?}

\section{Data sources}

Studies were identified by searching Medline (19662000), CINAHL (to 2000), SCI and SSCI (1969-2000), EMBASE/Excerpta Medica (1980-2000), the Cochrane Controlled Trials Register, CAB-Health (1973-97), HealthSTAR (1975-97); handsearching podiatry journals; reviewing bibliographies of reviews; and by contacting pharmaceutical companies and podiatry schools.

\section{Study selection}

Studies were selected if they were randomised controlled trials evaluating oral treatment in patients with clinically diagnosed and microscopy and laboratory confirmed tinea pedis.

\section{Data extraction}

2 reviewers independently extracted data on study design, condition, intervention, patient characteristics, study quality, and outcomes. The main outcome measure was mycological cure (negative results on microscopy and no dermatophyte growth in culture). Secondary outcomes were such clinical signs as redness, scaling, pustules, itching, burning, soreness; recurrence of the condition after achieving cure; and side effects.

\section{Main results}

12 trials were included and evaluated 5 antifungal treatments. 2 trials ( 1 of terbinafine and 1 of itraconazole) were placebo controlled; 9 trials compared different oral drugs (terbinafine, itraconazole, ketoconazole, fluconazole, and griseofulvin); and 1 trial compared different doses of fluconazole. Both active drugs were more effective than placebo: terbinafine, $250 \mathrm{mg}$ /day for 6 weeks, had a $65 \%$ (95\% CI 45 to 86$)$ absolute benefit increase (ABI) 2 weeks after the end of treatment. Itraconazole, $400 \mathrm{mg}$ /day for 1 week, had an ABI of 47\% (CI 29 to 67) 8 weeks after the end of treatment. Among the 9 trials comparing different drugs, terbinafine was no better than itraconazole in 3 trials, but 1 trial showed a benefit in favour of terbinafine (ABI 32\%, CI 16 to 47); itraconazole (1 trial) and ketoconazole (1 trial) did not differ from fluconazole; and griseofulvin did not differ from ketoconazole ( 1 trial). 2 trials comparing griseofulvin, $500 \mathrm{mg}$ /day, with terbinafine, $250 \mathrm{mg} /$ day, for 4 or 6 weeks, showed a higher cure rate with terbinafine (pooled ABI 52\%, CI 33 to 71). 1 trial comparing fluconazole, $50 \mathrm{mg} /$ day for 6 weeks, with $150 \mathrm{mg}$ /week for 6 weeks, showed no difference in cure rates. All trials reported short term side effects, including diarrhoea and nausea (most common); headache; and rash, dermatitis, and pruritus.

\section{Conclusions}

In patients with fungal infections of the skin of the foot, terbinafine or itraconazole eradicate infection better than placebo, and terbinafine is better than griseofulvin. Different azoles and different doses of fluconazole do not differ in effectiveness.

\section{COMMENTARY}

Currently, the selection of treatment for tinea pedis is muddied by the availability of both topical and systemic antifungal drugs. In this systematic review of oral treatments for tinea pedis, Bell-Syer $e t$ al note that topical treatment may be the realistic first line treatment plan.

Topical allylamines have been shown to produce a faster response than azoles, but the cure rates are similar. ${ }^{1}$ If the cost and availability of the products are similar, which is currently the case in the US, terbinafine, as an allylamine, may be preferable. Is terbinafine also the preferable treatment when oral treatment is required? Terbinafine was superior to griseofulvin in achieving mycological cure and has the advantage of healing more quickly. When studied head to head with itraconazole, higher cure rates were reported for terbinafine, but only 1 of 4 studies showed a statistically significant difference.

The drawbacks are, of course, cost and side effects. In the US, a 2 week course of terbinafine, $250 \mathrm{mg}$ /day, or itraconazole, $100 \mathrm{mg}$ /day, costs about US $\$ 100 .^{2}$ Compare this with the cost of topical treatment with azoles or allylamines at about $\$ 14$ for 1 week of treatment. Frequent side effects of systemic treatment include diarrhoea and nausea. However, no long term side effects were reported in any of the studies included in the review. Overall, terbinafine, and perhaps other allylamines, are more effective than azoles, but not dramatically so. This pokes more holes in the commonly held perception that terbinafines in all forms are superior to azoles in treating tinea pedis. ${ }^{3}$ Future work needs to address the comparative effectiveness of topical and oral treatments for tinea pedis.

Gene Elizabeth Harkless, RN, ARNP, DNSc Associate Professor and Family Nurse Practitioner University of New Hampshire, Department of Nursing Durham, New Hampshire, USA

1 Crawford F, Hart R, Bell-Syer S, et al. Topical treatments for fungal infections of the skin and nails of the foot. Cochrane Database Syst Rev 2002;(2):CD001434.

2 Database Syst Rev 2002;(2):CD001434. Drugstore.com (http://www.drugstore.com/pharm
prices/drugprice.asp?ndc=50458029004\&trx=1Z5006). prices/drugprice.asp?ndc=50458029004\&trx $=125006$ ). matic clinical trial is now needed. BMJ 1999;319:1070-1. 